\title{
Intensidad del fondo del cielo como indicador de eyecciones coronales solares y efectos geomagneticos
}

\author{
Maribel Suyapa Guerrero*, Guillermo Stemborg ${ }^{* *}$, Marta Roviera***
}

\section{RESUMEN}

Dada la importancia del impacto de las perturbaciones de los fenómenos geoefectivos en la magnetósfera de la Tierra y sus consecuencias sobre ella, resultan de mucho interés el estudio de todos los fenómenos solares que influencian el clima de la Tierra; en este caso las eyecciones coronales de masa solar (CME) a través de los datos del dispositivo Sky Tester del coronógrafo MICA (Mirror Coronograph in Argentina), un telescopio solar con base en Tierra, del coronógrafo C1 de LASCO a bordo del SOHO.

En esta investigación se ha planteado una correlación entre las CME como indicador del fondo de cielo a través de las mediciones del Sky Tester que observa el brillo del cielo alrededor del disco solar o aureola, llamado halo. También se plantea la correlación entre los halos CME y los disturbios geomagnéticos por medio del índice geomagnético "K", que indica el tipo de tormentas que provocan las CME sobre la magnetósfera terrestre.

Palabras claves: Eyecciones coronales de masa (CME), Halo, Coronógrafo, Índice $\mathrm{K}$, magnetósfera, disturbios geomagnéticos

\section{ABSTRAC}

Because of the importance of the impact of geoeffectiveshocks phenomena in Earth's magnetosphere and its impact on it, are of great interest the study of all solar phenomena that influence the Earth's climate, in this case the coronal ejections solar mass (CME) through the device data Tester Sky coronagraph MICA (Mirror coronograph in Argentina), a solar telescope and ground-based coronagraph LASCO-C1 aboard SOHO.

\footnotetext{
* Universidad Nacional Autónoma de Honduras. Facultad de Ciencias Espaciales. Escuela de Astronomía y Astrofísica. marsy3000@yahoo.com

** NASA/SOHO/LASCO INSTRUMENTS. stenburg@linpim.mgp.de

*** Instituto de Astronomía y Física del Espacio, IAFE, Argentina. rovira@iafe.uba.ar
} 
In this research has proposed a correlation between the CME as an indicator of sky background through Sky Tester measurements observing the brightness of the sky around the solar disk called halo. We also found evidence of the correlation between the halo CME and geomagnetic disturbances through geomagnetic index "K", which indicates the type of storms that cause CMEs on the Earth's magnetosphere.

Keywords: Coronal mass ejections (CME), Halo, Coronagraph, $\mathrm{K}$ Index, magnetosphere, geomagnetic disturbances 


\section{INTRODUCCIÓN}

Dada la importancia en la conexión Sol -Tierra el impacto de las perturbaciones de los fenómenos geoefectivos en la magnetósfera de la Tierra y sus consecuencias sobre ella, resulta de mucho interés el estudio de todos los fenómenos solares que influencian el clima de nuestro planeta; en este caso el estudio de las eyecciones coronales de masa solar (CME) utilizando los datos del coronógrafo MICA (Mirror Coronograph in Argentina), un telescopio solar con base en Tierra. Más aun con los indicios que se tienen, que con el pequeño dispositivo auxiliar Sky Tester del coronógrafo MICA se pueden predecir fenómenos solares. Aquí se trata de establecer un método que correlacione las eyecciones coronales de masa (CME) con las mediciones de ese dispositivo.

Con los sucesivos experimentos, han surgido nuevas preguntas y al mismo tiempo una mejor comprensión de la actividad solar y las CME. Es así que con el telescopio LASCO a bordo del SOHO, con su perfeccionada sensibilidad y resolución espacial, continúa esta tendencia. Se sabe que las CME se correlacionaron tempranamente con la ocurrencia de tormentas geomagnéticas (Gosling et al.1974). Estas tormentas causan diferentes clases de daños biológicos en seres humanos, animales y vegetales, sistemas de potencia, operaciones de naves espaciales, navegación aérea y líneas de conducción de petróleo, entre otros. Durante las tormentas extremas, se han visto auroras hasta en latitudes de 40. en Florida y el sur de Texas. Mientras la dinámica de estructuras eruptivas, individuales o eyectas aún no es bien conocida por medio de observaciones, parece determinante la velocidad inicial de una CME cercana al Sol para que un evento con dirección a la Tierra se convierta en una gran perturbación geomagnética.

Nuestro planeta está protegido de efectos dañinos de la radiación y del plasma caliente por la atmósfera y por una invisible cáscara magnética que desvía el plasma en el espacio; conocida como la magnetósfera. Esta es el resultado del campo magnético de la Tierra. (Ver figura 1). Una lluvia de plasma, dentro de nuestra magnetósfera, puede convertirse en tormenta magnética; 0 sea que perturba y penetra el campo magnético de la Tierra, comprobado con la aparición de las auroras, hasta en bajas latitudes en casos severos y extremos cualificados de acuerdo a un Índice geomagnético "K". Las tormentas se han clasificado en distintas categorías, de acuerdo al índice "K", que mide la desviación del disturbio de la componente más horizontal del campo magnético, observado sobre un magnetómetro y comparado con un día "quieto", para indicar el nivel de actividad de los tres días siguientes en tormentas magnéticas: 


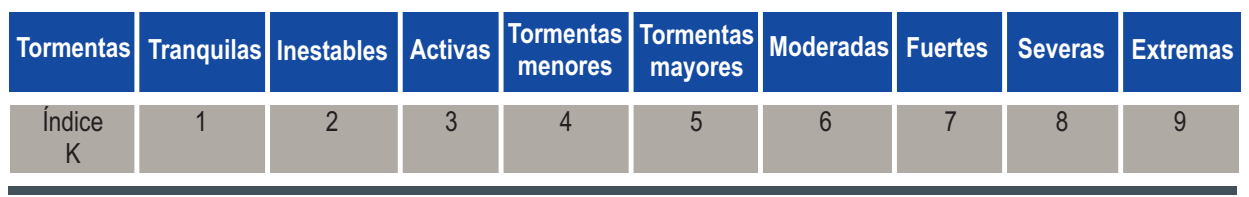

Lo antes expuesto refuerza este proyecto de investigación que presenta el análisis de dos correlaciones, una entre las CME y las mediciones que hace el coronógrafo Sky Tester por medio de la evaluación y análisis estadístico de las curvas de esas mediciones, en las que se sospechó alguna posible detección de una CME, y la otra entre las Halo CME y los disturbios en la magnetósfera de la Tierra, encontrando los índices geomagnéticos "k", que miden la desviación del campo magnético correspondientes a cada evento.

Figura 1. Campo magnético de la Tierra y Cinturón de radiación de Van Allen

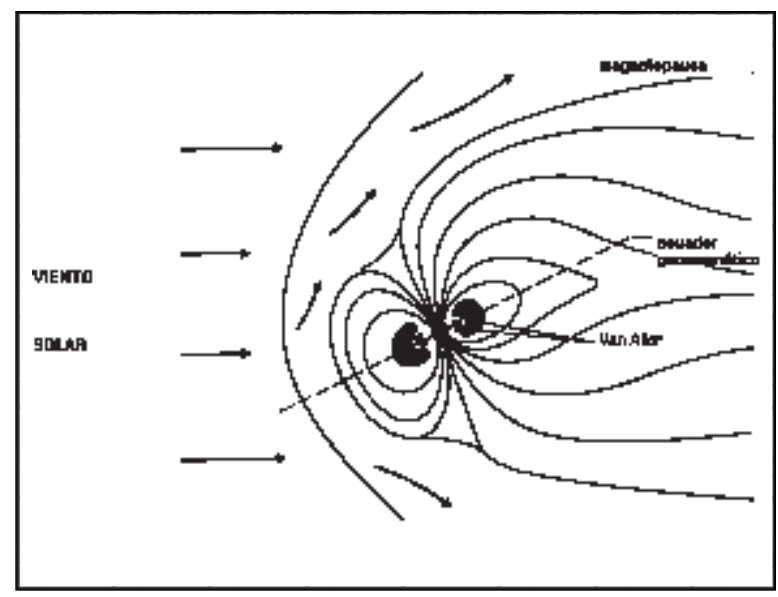

\section{METODOLOGÍAYEQUIPO}

Se realizo un conjunto de observaciones, estudios y análisis estadísticos de los datos con el fin de predecir las tormentas geomagnéticas y con el propósito de comprobar que los aumentos y caídas repentinas en la intensidad del cielo, denominados en esta investigación "Picos" medidos por el Sky Tester, son los indicadores de la ocurrencia de una CME observada en Tierra, planteada como la primera correlación. Los datos de este coronógrafo, ubicado en El Leoncito, San Juan, Argentina, correspondientes a un período de cuatro años, desde enero 1998 hasta junio 2001, en promedio unos cinco mil datos por día, fueron proporcionados 
en CD por el IAFE. Con esta información se buscaron eyecciones coronales de masa, con dirección a la Tierra, en el sitio web http://lascowww.nrl.navy.mil/cmelist.html de LASCO de ese período para ser estudiados; para comparar las curvas de los eventos, se revisó la página web de LASCO MPAe, que muestra esta información. Finalmente, para una mejor interpretación de las curvas, se generaron los gráficos en los software especiales para los casos con posibilidad de correlación entre CME y las observaciones del Sky Tester.

\section{Equipo}

Para la concreción de los objetivos buscados se utilizaron los datos provistos por varios instrumentos:

a) Los instrumentos auxiliares denominados Sky y Sun Tester del coronógrafo de espejo MICA (por sus siglas en ingles: Mirror Coronagraph for Argentina).

b) El coronógrafo LASCO-C2 a bordo del Observatorio Solar y Heliosférico SOHO (por sus siglas en inglés Solar and Heliospheric Observatory).

\section{El coronógrafo}

El coronógrafo es un instrumento óptico diseñado por el astrónomo francés Bernard Lyot con el fin de observar la tenue corona solar en el rango visible del espectro de radiación, en ausencia de un eclipse solar total. Dentro del coronógrafo la imagen del Sol es eclipsada artificialmente por medio de un disco que, según la parte de la óptica donde se coloque, determinará el tipo de coronógrafo: "interno" si el disco se coloca detrás del objetivo, y "externo" si se coloca por delante. La clave de un coronógrafo es reducir al mínimo la luz dispersada dentro del instrumento. A fin de optimizar la reducción de luz dispersada dentro del instrumento, la elección del sitio donde el coronógrafo vaya a ser instalado es de fundamental importancia. Para reducir la dispersión provocada por la atmósfera terrestre, se suelen elegir lugares secos y a grandes alturas. El caso ideal es a bordo de satélites, donde los efectos ocasionados por la atmósfera terrestre desaparecen. Abajo la figura 2 muestra un coronógrafo

Figura 2. Coronógrafo

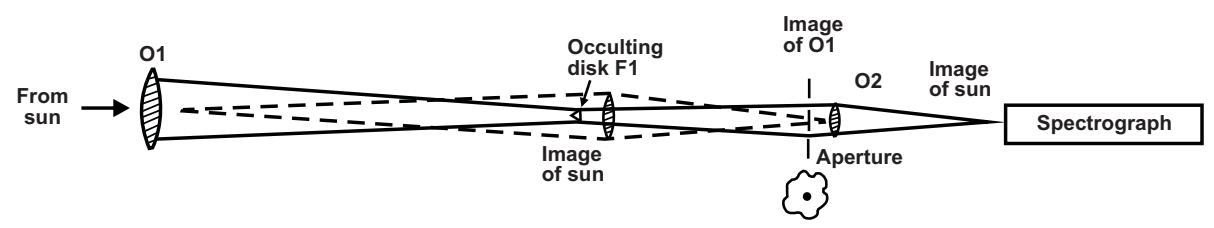


El MICA (Mirror Corograph for Argentina) es un telescopio solar con base en tierra, forma parte de un convenio de cooperación entre Argentina y Alemania. Por Argentina participan el IAFE (Instituto de Astronomía y Física del Espacio) y el OAFA (Observatorio Astronómico Félix Aguilar), por Alemania participan el MPAe (Max Planck Institute fuer Aeronomy) y el MPE (Max Planck Institute fuer Extraterrestrische Physik). El MICA actualmente se encuentra funcionando en la estación de altura Carlos Ulrico Cesco, en El Leoncito, San Juan, Argentina, a 2400 m sobre el nivel del mar a $69.30 \mathrm{~W}$ de longitud y $31.80 \mathrm{~S}$ de latitud. MICA es un coronógrafo de espejo con ocultador interno. Es un Instrumento Lyot con un set completo de espejos y lentes y sistemas electrónicos y mecánicos que permiten el funcionamiento en modo automático del mismo. MICA es un coronógrafo avanzado que complementa las mediciones del LASCO. Está dedicado al estudio de eventos dinámicos en la corona solar, en la conocida línea verde coronal (línea Fe XIV a $530.3 \mathrm{~nm}$ ), con énfasis en observaciones de fenómenos transitorios y estudios de la estructura y evolución de prominencias solares, entre otros.

El telescopio MICA tiene dos dispositivos auxiliares: el Sky Tester y el Sun Tester ubicados muy cerca del MICA; El primero observa el brillo del cielo alrededor del disco del Sol o aureola. Es muy sensible a las nubes. El SunTester mide la intensidad del disco del Sol donde el valor más alto es el brillo más intenso del disco solar. El nivel de intensidad registrado por ambos instrumentos es función de la masa de aire, así que la señal del Sun Tester en el zenit local se espera que sea baja, mientras la señal es máxima en el Sky Tester.

Figura 3. Dispositivo Sky Tester. Coronógrafo refractivo con ocultador externo.

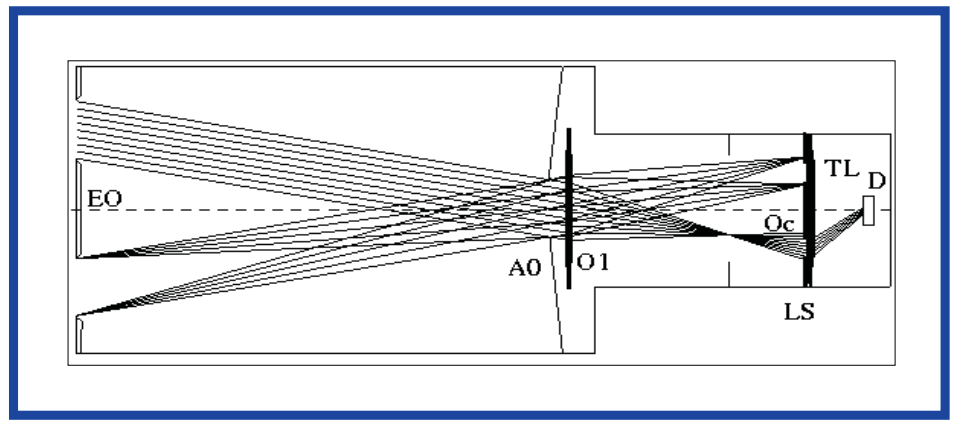

E0: Ocultador externo

A0: Apertura de entrada.

01: Lente Objetivo

Oc: Ocultador Interno
LS: Lyot stop

TL: telelentes

D: detector (plano focal) 
Las mediciones se hacen en promedio cada cinco segundos. Estos valores son usados por un software de control del telescopio que junto con la medición de la velocidad del viento, obtenida por una estación de clima automáticamente, decide si las condiciones son buenas para observar la corona solar, iniciando o parando la rutina de observación según sea el clima. La intensidad medida por los dispositivos auxiliares del telescopio MICA, Sky y Sun Tester, es medida en mili voltios que se grafican en el eje de las ordenadas y el tiempo en el eje de las abscisas. Las unidades para el tiempo son horas y minutos del tiempo universal UT (por sus siglas en inglés Universal time). Los datos se publican en forma gráfica (Ver Fig 4) en la página del telescopio MICAen internet. Los gráficos que no se encontraron en el sitio de MICA, se hicieron con un software especial.

Figura 4. Gráfico publicado por la página web del MICA.

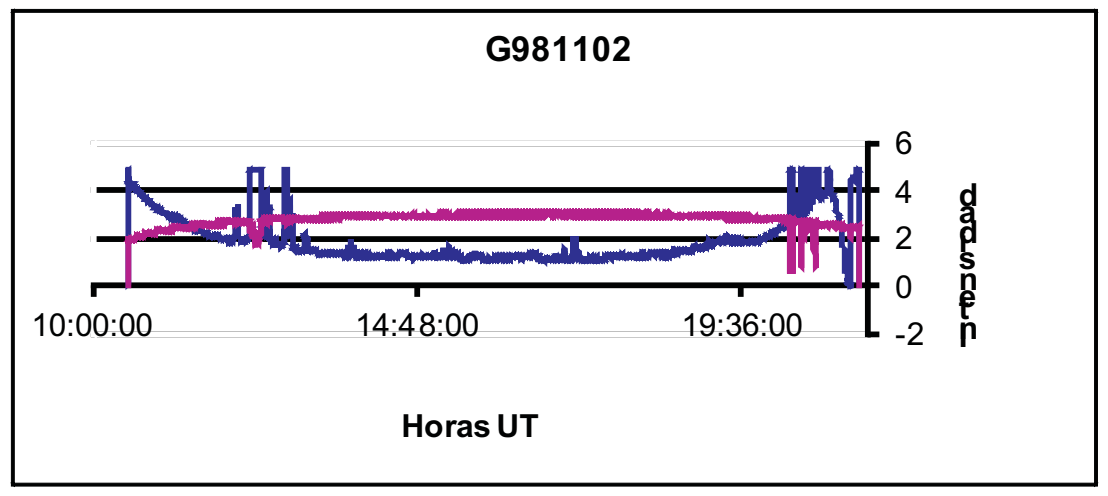

\section{LASCO y SOHO}

EI LASCO (Large Angle and Spectrometric Coronagraph) es uno de doce instrumentos a bordo de la nave espacial $\mathrm{SOHO}$, lanzada por cooperación entre la ESA y la NASA. SOHO fue lanzado el 2 de diciembre de 1995 desde el Centro Espacial Kennedy, Cabo Cañaveral, Florida.

LASCO está formado por un conjunto de tres coronógrafos que registran imágenes de la corona en luz blanca, desde 1.1 hasta 32 radios solares. (Un radio solar es aproximadamente 700,000 km., 420,000 millas ó 16 minutos de arco). LASCO incluye tres coronógrafos: el C1, C2, y C3 con campo de visión circular. El C1 es un coronógrafo reflectivo, con ocultador interno, mientras que C2 y C3 son coronógrafos con ocultador externo. Una descripción detallada de LASCO se encuentra en Brueckner et al., 1995, Solar Physics, 162, 357. LASCO y EIT proveen 
a SOHO con la capacidad de fotografiar la corona solar desde su base a través de 30 radios solares. El C1 observa de 1.1 a 3 radios solares, el C2 observa de 1.5 a 6 radios solares, el C3 observa de 3.7 a 30 radios solares.

Para llevar a cabo los objetivos propuestos en esta tesis, se obtuvo de la página de LASCO la información complementaria que confirma la ocurrencia de las eyecciones coronales de masa, con datos de fecha, hora, dirección, entre otros datos. Cabe mencionar que el tiempo de duración de cada evento se calculó de los videos correspondientes. Las imágenes (Fig. 5) muestran algunos eventos observados por el coronógrafo C2 y C3.

Figura 5. Imágenes reales de la corona del Sol con fecha y hora. Del C2 de LASCO ( a la izquierda): Eyecciones coronales de masa solar y un cometa pasando del C3 (derecha) eyecciones coronales de masa solar
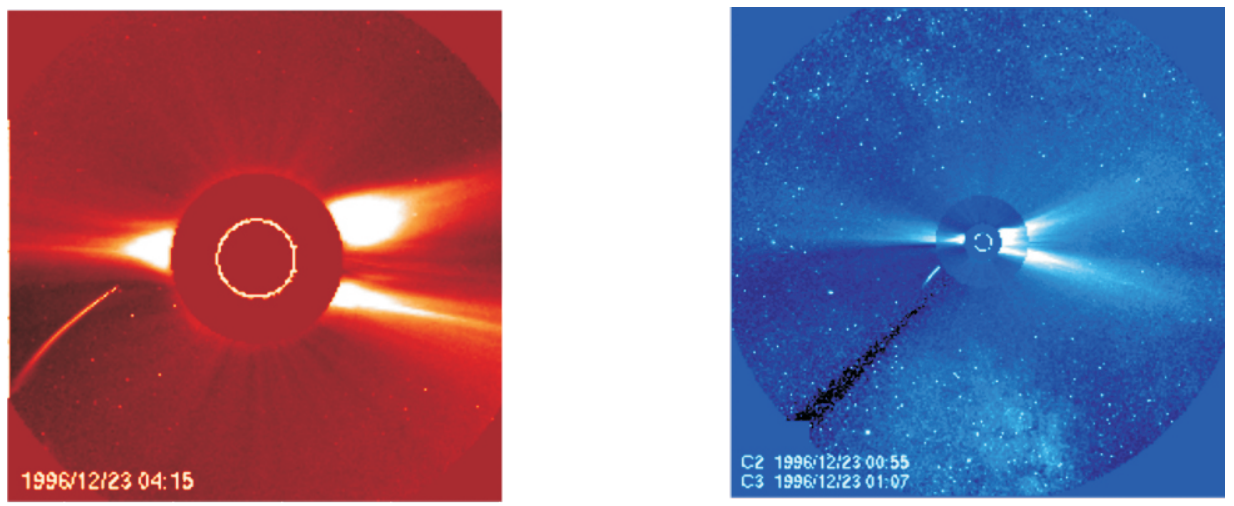

\section{ANÁLISIS DE DATOS E INTERPRETACIÓN}

Previo al análisis de datos se hizo la lectura y organización de estos. Se continuó con un despliegue y lectura de los datos del CD en Excel. Con este arreglo se crearon varios archivos de diferente tipo, creados para hacer las aproximaciones estadísticas y gráficos respectivos.

Se buscaron en la lista de eyecciones coronales de masa (CME List)de LASCO, los eventos con dirección a la Tierra para ese período, de la que se obtuvieron 10 eventos para el año 1998, 10 para 1999, 38 para el 2000 y 41 para el 2001, haciendo un total de 99 eventos (lista1)(ver anexo A3). De estos se eliminaron 52 casos que no concuerdan con la rutina de horario de observación del MICA, quedando 47. 


\section{Obtención de rangos de tiempo para cada CME}

Los rangos de tiempo de ocurrencia de los fenómenos observados por el telescopio MICA, desde enero 1998 a junio 2001, se obtuvieron de la siguiente forma: El tiempo inicial es la hora y minutos en que el coronógrafo C2 de LASCO mira por primera vez la CME. Así es que los tiempos iníciales se tomaron de la "Coronal Mass Ejection List", la cual reporta una lista de observaciones en luz blanca de los eventos más grandes de LASCO, dividida por años.

Mientras que el tiempo final de cada CME se obtuvo observando el video que corresponde a la fecha de cada evento, cocando el cursor en el video a partir de la hora de inicio del evento hasta llegar al momento en que la CME sale del campo de visión del coronógrafo C2 de LASCO, en el cual podemos apreciar el tiempo en horas y minutos que corresponde al tiempo final de la CME que nos ocupa. Se buscaron los datos dentro de cada rango de tiempo para ser analizados estadísticamente y, consecuentemente, hallar los "picos" más cercanos a la hora de ocurrencia de cada CME. Las primeras estadísticas se tomaron de los datos crudos de 40 casos de las intensidades del Sky y Sun Tester, para ser comparadas con las estadísticas finales de aproximación.

\section{Curvas de aproximación y estadísticas}

Partiendo de la colección de datos arriba mencionada, se ordenaron en tres archivos uno con los pares ordenados $\mathrm{X}$ e $\mathrm{Y}$, en un sistema de coordenadas rectangulares, que corresponden a Tiempo e Intensidad medida por el dispositivo Sky Tester. De este formato se desprenden otros dos archivos: el de tiempo usado por el Sky Tester, que es de horas y minutos (h: $\mathrm{m}$ ), para crear las curvas de los datos reducidas en el rango de tiempo como también las curvas de aproximación lineal. Y el otro archivo es el correspondiente a la intensidad del cielo alrededor del disco del Sol (halos). Obtenidos los rangos de tiempo (tiempo inicial - tiempo final) y las intensidades reales ( $Y$ real), medidas por el Sky Tester, se hicieron ecuaciones matemáticas, usando el programa "El Matemática", que expresan la relación entre estas dos variables por el método de mínimos cuadrados, que se utiliza para evitar juicios individuales en la construcción de cualquier curva de aproximación para ajustarse a colecciones de datos y se conoce como la mejor curva de ajuste.

Primero se calcularon las curvas para ecuaciones lineales y para ecuaciones de segundo grado, para ver la diferencia de cada una con la curva real. Al hacer las estadísticas se comprobó que la diferencia existente entre las curvas lineales con las de segundo grado es despreciable. Se continuó el análisis de datos gráficamente, se 
plasmaron en todos los casos las curvas de aproximación lineales y de segundo grado, para comparar los datos reales con las tendencias ideales de las curvas de aproximación. Aunque la mejor aproximación para los datos de Sky Testees una curva exponencial, porque cuando se inicia la observación, esta parte de la curva es creciente; al final de cada observación la curva también es decreciente. Como primera aproximación se desprecia que la curva sea exponencial, entonces tomamos un rango de tiempo antes del pico y después del pico; en que el comportamiento de los valores es lineal.

En este análisis se usan ecuaciones de primer grado para aplanar este tramo de la curva de los datos reales, a los cuales se les ha calculado su promedio, mediana y desviación estándar. Se hicieron las curvas reducidas para los 47 eventos, quedando en este paso $8 \mathrm{CME}$, para hacer aún más reducido el rango de tiempo y observar la variación en la intensidad, que muestran los saltos como posibles picos.

\section{RESULTADOS}

De los aproximadamente 6.3 millones de datos obtenidos por el Sky Tester para el período estudiado, se encontraron 10 eventos para el año 1998, 10 para 1999, 38 para el 2000 y 41 para el 2001, haciendo un total de 99 eventos. De estos se eliminaron 52 casos que no concuerdan con la rutina de horario de observación del MICA, quedando 47 para ser estudiados. Para comparar las curvas de los 47 eventos, se revisó la página web de LASCO MPAe, aunque finalmente para una mejor interpretación de las curvas, siempre se generaron todos los 47 casos con posibilidad de correlación entre CME y las observaciones del Sky Tester. En la Tabla I observamos los picos encontrados numéricamente en las curvas que se analizaron primero en forma visual y después matemáticamente.

Tabla I. Eventos con "Picos" más representativos en la seňal

\begin{tabular}{|c|c|c|c|c|c|}
\hline Fecha & $\begin{array}{c}\text { Intensidad } \\
\text { del cielo } \\
\text { (Sky Tester) }\end{array}$ & Promedio & Mediana & Desv. Estand & $\begin{array}{c}\text { P+3 Desv. } \\
\text { Estand }\end{array}$ \\
\hline 25/01/1998 & 2.10 & 0.0238347 & 0.00955706 & 0.0309828 & 0.32 \\
\hline $06 / 11 / 1998$ & 1.945 & 0.0056411 & 0.00421517 & 0.00537397 & 0.021 \\
\hline $05 / 09 / 2000$ & 1.66 & 0.0717459 & 0.0589738 & 0.0583165 & 0.0246 \\
\hline $05 / 02 / 2001$ & 2.60 & 0.000239305 & 0.000145813 & 0.000313882 & 0.000676 \\
\hline
\end{tabular}

* El concepto es que si le sumamos y restamos a la media 3 veces la desviación estándar, obtenemos el intervalo en el que están contenidos el $99.9997 \%$ de los datos. 
Tabla I. Resultados

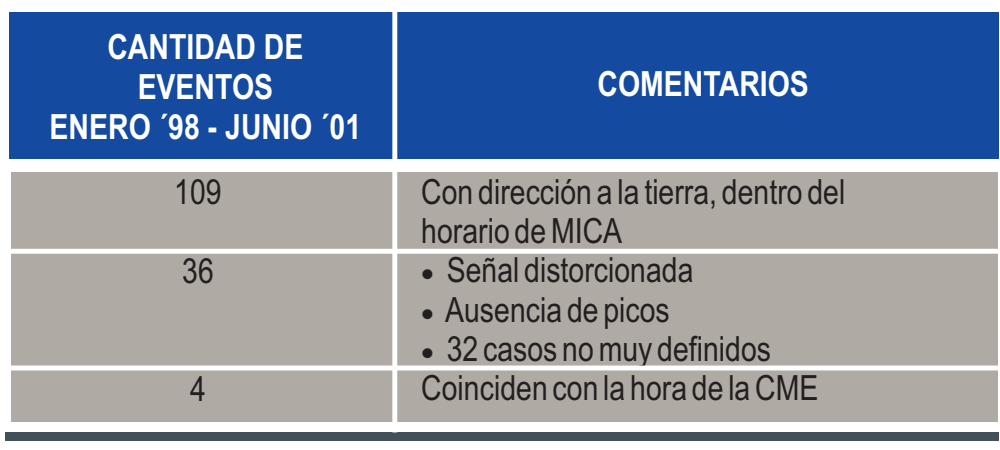

De la Tabla I se puede concluir que los cuatro casos de eyecciones coronales de masa presentan: las estadísticas de la intensidad medida por el Sky Tester: donde se ve que todas las intensidades de cielo medidas por el Sky Tester son mucho mayor que el promedio más 3 veces la desviación estándar (Intensidad i > Pi+3DSi).

Estos datos estadísticos nos dicen que los PICOS gráficos son verdaderamente observaciones del Sky Tester de CME confirman esta propuesta la exacta coincidencia de las horas de ocurrencia de las eyecciones coronales de masa, reportadas por el C2, con los PICOS gráficos. Ver ej. siguientes:

GRÁFICOS: Observaciones REALES de MICA

No. 1. Gráfico 980125

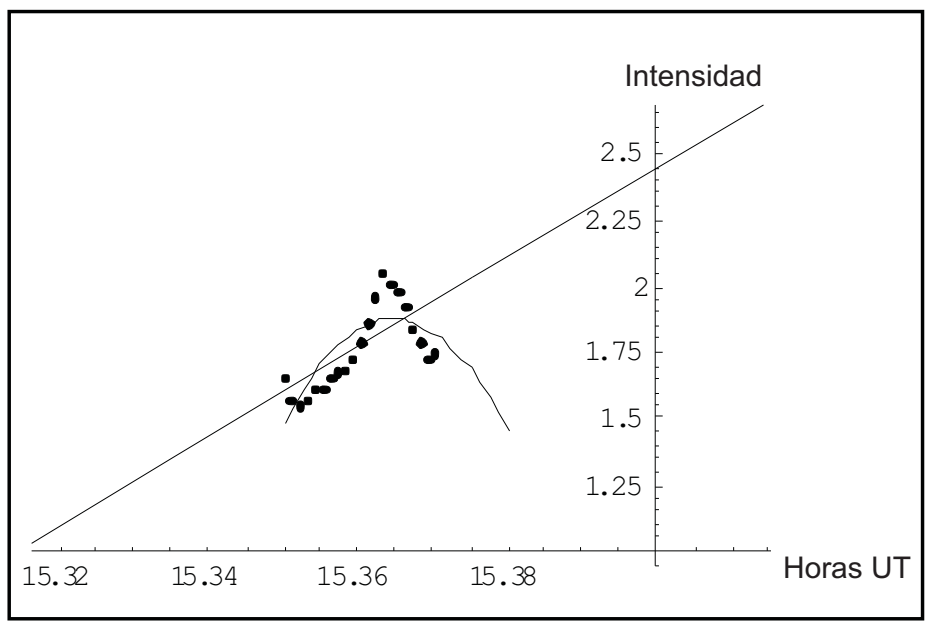

Halo CME / Pico: $(15.36,2.10)$ 
No. 2. Gráfico 981106

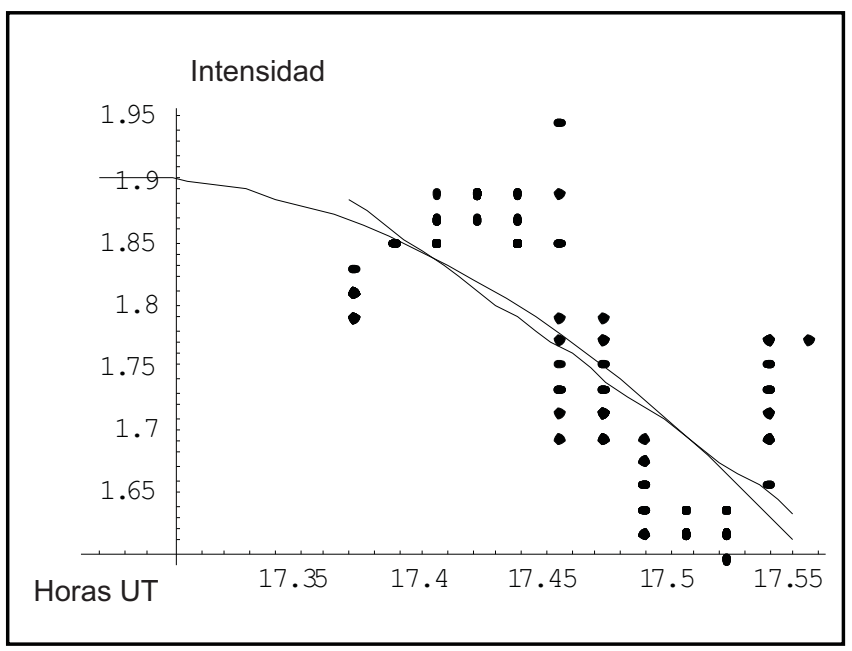

CME/ Pico $(17.45,1.945)$

Intensidad (mili voltios) Intensidad (mili voltios)

No. 3. Gráfico 000905

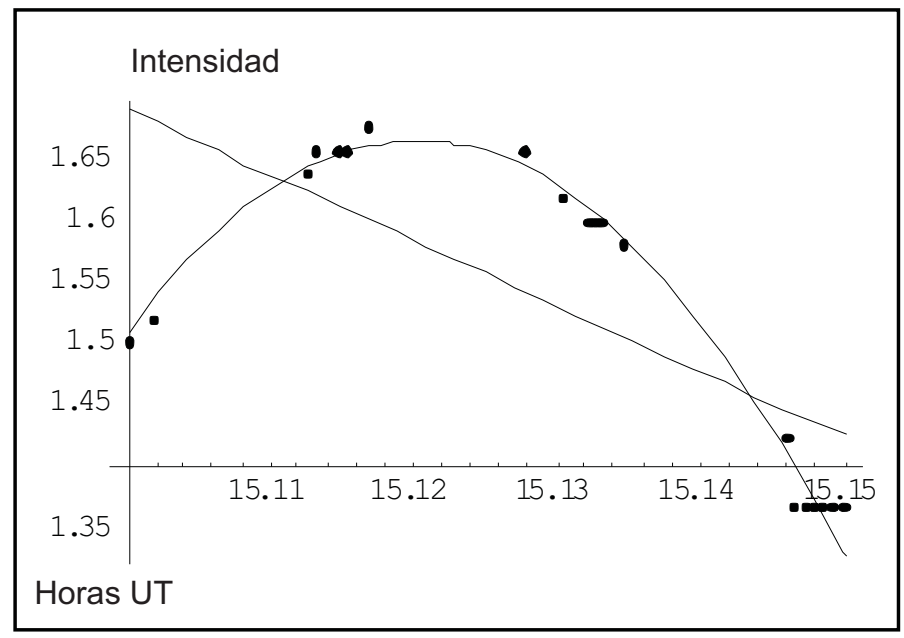

Por otra parte se sabe que el Sky Tester observa de 1.05 R.S hasta 2R.S y que el coronógrafo C2 lo hace desde 1.5 a 6 R.S, por lo que se esperaba que el Sky Tester detectara los eventos antes que el $\mathrm{C} 2$, pero hemos encontrado por medio del análisis gráfico que ambas observaciones son simultáneas. De los gráficos 980125 , 981106, 000905 y 010205 se concluye que el Sky Tester ha detectado las CME, cuando no hay perturbaciones atmosféricas en el sitio de observación. Utilizando 
como parámetros significativos la velocidad y la aceleración de las CME para documentar cada caso de las CME con dirección a la Tierra, por ejemplo vemos que la CME del 000905 presenta baja velocidad, con un frenado alto de $24 \mathrm{~m} / \mathrm{s}^{\wedge} 2$ y buenas condiciones atmosféricas, mostradas estas últimas gráficamente por el dispositivo Sun Tester. Refuerzan esta primera propuesta del proyecto, para la correlación de CME con las mediciones del Sky Tester, las distancias recorridas por las eyecciones coronales de masa encontradas a partir de su velocidad y se ha demostrado que todas están dentro del rango de observación del Sky Tester:

$$
34,813.25 \mathrm{~km}<\mathrm{D}<661,451.75 \mathrm{~km} \text {. }
$$

Cálculo del rango de distancia:

Sabemos que 1 R.S $=696,265 \mathrm{~km}$

Se toma el 5\% dado por el Sky Tester,(de 1.05 R.S) $=34,813.25 \mathrm{~km}$. $696,265 \mathrm{~km}-34,813.25 \mathrm{~km}=661,451.75 \mathrm{~km}$.

Otro factor importante en esta propuesta son las estadísticas de los datos crudos producidos por el Sky Tester (Tabla 1) demostrando que los picos gráficos del Sky Tester representan un salto repentino en la intensidad del cielo alrededor del disco que ha sido detectado por el coronógrafo Sky Tester, pero que observa menos de 12 horas diarias. Con la primera investigación del estudio de los datos antes mencionados se asume una posible correlación entre las observaciones del Sky Tester y los Halo CME, la cual puede ser confirmada con un estudio más detallado.

Tabla III. Resumen Índices "K"

\begin{tabular}{|c|c|c|c|}
\hline No. & FECHA & FENÓMENO & (d/h/min), Índice "K" \\
\hline 1 & 25/01/1998 & HALO PARCIAL & $28 / 20: 30, K=7$, tormenta fuerte \\
\hline 2 & $11 / 05 / 1998$ & HALO PARCIAL & $314 / 25: 24, K=8$, tormenta severa \\
\hline 3 & 02/11/1998 & HALO PARCIAL & 307/10:27, K=7, tormenta fuerte \\
\hline 4 & 06/11/1998 & HALO PARCIAL & $314 / 25: 24, K=9$, tormenta extrema \\
\hline 5 & $11 / 03 / 199$ & HALO PARCIAL & $74 / 03: 58, \mathrm{~K}=10$, tormenta extrema \\
\hline 6 & $18 / 02 / 2000$ & FULL HALO & $52 / 17: 30, \mathrm{~K}=10$, tormenta extrema \\
\hline 7 & $06 / 04 / 2000$ & HALO PARCIAL & $96 / 02: 45, K=9$, tormenta extrema \\
\hline 8 & $16 / 06 / 2000$ & HALO PARCIAL & 171/02:05, K=7, tormenta fuerte \\
\hline 9 & 05/09/2000 & HALO PARCIAL & $251 / 10: 27, K=9$, tormenta extrema \\
\hline 10 & $25 / 10 / 2000$ & FULL HALO & $302 / 12: 22, \mathrm{~K}=10$, tormenta extrema \\
\hline 11 & $02 / 11 / 2000$ & HALO & $307 / 14: 49, \mathrm{~K}=7$, tormenta fuerte \\
\hline 12 & 09/02/2000 & $\mathrm{HALO}$ & $43 / 17: 04, K=10$, tormenta extrema \\
\hline 13 & $10 / 02 / 2000$ & HALO & 44/16:37, $K=10$, tormenta extrema \\
\hline 14 & $14 / 02 / 2000$ & FULL HALO & 90/20:00, K=10, tormenta fuerte \\
\hline 15 & $14 / 04 / 2000$ & HALO PARCIAL & $44 / 16: 37, K=10$, tormenta extrema \\
\hline
\end{tabular}


Por otra parte, de la Tabla III la segunda correlación entre Halos CME y disturbios geomagnéticos se ha encontrado a partir del índice "k", que indica la máxima desviación del disturbio, del componente más horizontal del campo magnético de la Tierra. En la tabla Il podemos observar que los casos sobresalientes en este estudio son todos halos parciales que produjeron tres categorías de tormentas: fuertes, severas y extremas, con índices "K" = 7, 9, 10. En ninguno de los casos estudiados se observaron tormentas menores con índice $" k "=5$, ni tormentas moderadas con índice "K"=6, ambas de menor impacto en la magnetósfera de la Tierra.

En resumen, lo antes mencionado nos da los primeros indicios de que la segunda hipótesis planteada sobre la correlación entre los HALO -CME y los disturbios geomagnéticos, están correlacionados dentro de la primera etapa de esta investigación. Estos primeros indicios se pueden confirmar igual que la primera correlación, con una investigación más detallada.

\section{DISCUSIÓN}

Con esta investigación, por un lado, se han cimentado los indicios de una posible correlación de eyecciones coronales de masa con las observaciones de Sky tester ya que los cuatro eventos más representativos arrojan magníficos datos estadísticos individuales, que no son suficientes para los 109 CME con dirección a la Tierra analizados; lo contrario hubiera sido si el resultado fuese al menos un tercio con esas estadísticas. En los resultados obtenidos en la correlación entre halos CME y disturbios geomagnéticos se ve que todos estos eventos produjeron tormentas magnéticas fuertes, severas y extremas con el índice geomagnético"K" de alerta, algo ya conocido y esperado.

\section{CONCLUSIONES}

La importancia de la física solar en los efectos sobre la Tierra mantiene abiertas amplias expectativas en la investigación de ese campo, con los resultados encontrados en esta tesis no se ha llegado a establecer conclusiones específicas en cuanto a las correlaciones buscadas; de las tablas resumen vemos que la mayor parte de datos se obtuvieron entre el año 2000 y 2001, o sea la época del último máximo de actividad solar, del ciclo de 11 años que también presentó baja actividad aunque se incrementó débilmente respecto a 1989. Aun así de 99 eventos se eliminaron 52 casos que no concuerdan con la rutina de horario de observación del MICA, quedando 47 en estudio. 
Cabe la hipótesis: puede ser que estos 52 eventos llegaron a la Tierra durante la noche, consecuentemente no pudieron ser observados por el telescopio solar MICA en Argentina, que tiene las limitantes de todos los telescopios con base en tierra.

Por lo que se sugiere continuar la investigación para confirmar la hipótesis, durante otras épocas de máxima actividad del Sol, en los que ocurre una gran cantidad de CME que se convierten en tormentas geomagnéticas. Porque vemos después del análisis que los aproximados 8 millones de datos de cuatro años, durante el día solar, no son suficientes, debido a que el día solar aparente (Sol sobre el horizonte) es de corta duración en esa latitud de San Juan en Argentina. Por lo tanto se necesitan muchos más datos de observación para análisis estadístico y técnico tendientes a una mejor evidencia de las correlaciones buscadas para el telescopio solar MICA.

\section{AGRADECIMIENTOS}

A la Dirección de Investigación Científica (DICU) de la UNAH y su cuerpo técnico, por el apoyo a los trabajos de investigación dentro de la Astrofísica Solar y Clima espacial, colaborando de esta manera con el crecimiento de este complejo y maravilloso campo del conocimiento en Honduras.

\section{REFERENCIAS BIBLIOGRÁFICAS}

Chen James,(2000), Coronal Mass Ejections, causes and consequences, Geophysical

Howard, R. A et Al, , Journal of Geophysical Research (ISSN 0148-0227), Vol 90,1985 Sept. 1,p. 8173-8191.

http://lasco-www.nrl.navy.mil/about_lasco.html

http://sohowww.nascom.nasa.gov/descriptions/mission/english/

http://star.mpae.gwdg.de/mica/mica_home.htm

http://www.sec.noaa.gov/weekly/weekly97.html

http;//Star.mpae.gwdg.de/mica/theinstrument/MICA-A.pdf

http;//www.sec.noaa.gov/ftpdir/list/hpi/power_1999.txt

Hundhausen, A. J, coronal mass ejections in cosmic winds and the heliosphere,eds. J.R. Jokipiiet al., Arizona, Tucson, in press, 1996.

Hundhausen, A.1999. The many faces of the sun: sumary of the results from NASA's

Solar Maximum Mission. Edited by Keith T. Strong, Julia L.R. Saba, Bernard M. Haisch, and Joan T. Schmelz. New York : Springer, 1999., p.143.

Koomen, M.J. et Al. 1978, Appliied Optics, Volume 17, Issue 15, August 1, 1978, 